\section{Discovering hypertriglyceridaemia}

To the Editor: Hypertriglyceridaemia is a well-established, but occasionally overlooked, cause of pancreatitis despite mention in South African (SA) guidelines over many years. ${ }^{[1,2]}$ Hypertriglyceridaemia of $>10 \mathrm{mmol} / \mathrm{L}$ is associated with a severe course of pancreatitis. ${ }^{[3]}$ Appropriate management of severe hypertriglyceridaemia can prevent acute pancreatitis, improve quality of life, reduce absenteeism, avoid expensive treatment of complications, such as acute respiratory distress and renal failure, and prevent premature death. A lipaemic blood sample indicates severe hypertriglyceridaemia and should prompt measurement of triglycerides. Responses to interventions vary widely, depending on controlling secondary causes that increase the production of triglyceride-rich lipoproteins (e.g. diabetes and excess alcohol intake), and on the lipoprotein lipase activity. In the absence of lipoprotein lipase activity, patients do not respond to fibrates that are generally recommended for hypertriglyceridaemia. There is no response to statins or ezetimibe. Restriction of dietary triglyceride intake to $<40 \mathrm{~g} /$ day is vital. New drugs ${ }^{[4]}$ or genetic strategies may become available.

In the public healthcare sector, there is limited scope for patients with severe hypertriglyceridaemia to attend specialised clinics. In the private healthcare sector, specialised expertise is rare. Reimbursement of lipid-lowering medication, controlled by medical schemes whose application forms capture clinical details and results of a lipogram, can assist clinicians by careful review of prescriptions and therapeutic outcomes for patients with severe hypertriglyceridaemia.

Personal experience with 3 cases of chylomicronaemia due to severely impaired lipoprotein lipase activity (Fredrickson type I hyperlipidaemia) demonstrates the plight of patients with uncommon, severe metabolic errors in SA. Recurrent pancreatitis due to severe hypertriglyceridaemia that had not responded to combination treatment of atorvastatin and gemfibrozil in a 43-year-old man, resulted in admission to an intensive care unit in 2005. The patient had pancreatitis complicated by respiratory failure. Weeks later he underwent surgery for a pancreatic pseudocyst. A low-fat diet alone controlled the dyslipidaemia and there have been no subsequent problems. The patient had an ideal lipid profile on a restricted fat diet alone. Senior members of his medical scheme were unsuccessfully approached to support special units to ensure optimal management of severe dyslipidaemias. More recently, the same medical scheme approved simvastatin and ezetimibe for severe hypertriglyceridaemia in a 44-year-old woman with genetically proven lipoprotein lipase deficiency in whom recurrent pancreatitis had resulted in diabetes and, along with hypertension, resulted in a stroke. A 60-year-old man with diabetes and proven lipoprotein lipase deficiency underwent placement of a coronary artery stent for angina pectoris. He sought advice on the approved management with atorvastatin together with bezafibrate. The chronic medication section of the same medical scheme was approached by email, with a request that a medical practitioner should explain the decision, as no direct contact details were found. After some additional communications, there was an undertaking to inspect the patient's records but no explanation has been forthcoming.

Severe hypertriglyceridaemia should be addressed urgently to avoid pancreatitis by dealing with secondary causes, restricting dietary fat intake and judicious prescription of medication. It is suggested that severely hypertriglyceridaemic patients be assessed at special (lipid) clinics linked to a dedicated laboratory, which can discern mono- and polygenic causes of chylomicronaemia that influence treatment options and prognosis. ${ }^{[5]}$ Such specialised centres, preferably at teaching hospitals, could be part of a National Health Insurance (NHI) plan to serve public and private sector patients alike. The patient's wellbeing is of the utmost importance and uncommon and rare disorders justify the creation of special centres. Such an arrangement will record the local experience, contribute to the training of medical practitioners and judiciously translate new developments into practice.

\section{A D Marais}

Division of Chemical Pathology, Department of Pathology, Faculty of Health Sciences, University of Cape Town, South Africa

david.marais@uct.ac.za

\section{J Blom}

Division of Lipidology, Department of Medicine, Faculty of Health Sciences, University of Cape Town, South Africa

1. Rossouw JE, Steyn K, Berger GMB, et al. Action limits for serum total cholesterol: A statement for the medical profession by an ad hoc committee of the Heart Foundation of Southern Africa. S Afr Med J 1988:73(12):693-700.

2. Klug E, Raal FJ, Marais AD, et al. South African dyslipidaemia guideline consensus statement: 2018 update. A joint statement from the South African Heart Association (SA Heart) and the Lipid and Atherosclerosis Society of Southern Africa (LASSA). S Afr Med J 2018;26;108(11b):973-1000. https://do org/10.7196/SAMJ.2018.v108i11.13383

3. Pascual I, Sanahuja A, García N, et al. Association of elevated serum triglyceride levels with a more sever course of acute pancreatitis: Cohort analysis of 1457 patients. Pancreatology 2019;19(5):623-629. https:/ doi.org/10.1016/j.pan.2019.06.006

4. Nurmohamed NS, Dallinga-Thie GM, Stroes ESG. Targeting apoC-III and ANGPTL3 in the treatmen of hypertriglyceridemia. Expert Rev Cardiovasc Ther 2020;18(6):355-361. https://doi.org/10.1080/1477 9072.2020 .1768848

5. D’Erasmo L, di Costanzo A, Cassandra F, et al. Spectrum of mutations and long-term clinical outcomes in genetic chylomicronemia syndromes. Arterioscler Thromb Vasc Biol 2019;39:2531-2541. https://doi org/10.1161/ATVBAHA.119.31340

S Afr Med J 2021;111(8):697. https://doi.org/10.7196/SAMJ.2021.v111i8.15783 